\title{
Dinâmica temporal do míldio da videira em variedades Piwi na região de San Michele all'Adige, Trentino - Itália
}

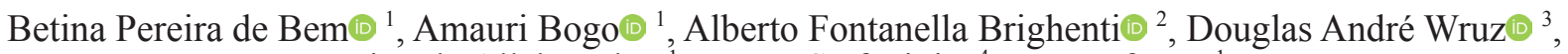 \\ Ricardo Allebrandt@ ${ }^{1}$, Marco Stefanini ${ }^{4}$, Leo Rufato ${ }^{1}{ }^{1}$
}

\begin{abstract}
${ }^{1}$ Universidade do Estado de Santa Catarina, CAV/UDESC, Av. Luiz de Camões, 2090 - Conta Dinheiro, CEP: 88520-000, Lages, SC, Brasil. ${ }^{2}$ Universidade Federal de Santa Catarina, CCA/ UFSC, Rod. Admar Gonzaga, 1346 - CEP: 88034-000, Itacorubi, Florianópolis, SC, Brasil. ${ }^{3}$ Instituto Federal de Santa Catarina, IFSC- Campus Canoinhas; Av. Expedicionários, 2150 - Campo da Água Verde, CEP: 89466-312, Canoinhas, SC, Brasil. ${ }^{4}$ Fundazione Edmund Mach, FEM ; Via Edmund Mach, 1, 38010 San Michele All'adige TN, Itália
\end{abstract}

Corresponding author: Betina Pereira de Bem (betadebem@yahoo.com.br)

Data de chegada: 15/10/2019. Aceito para publicação em: 15/03/2020.

$10.1590 / 0100-5405 / 230013$

\section{RESUMO}

Bem, B.P.; Bogo, A.; Brighenti, A.F.; Wruz, D.A.; Allebrandt, R.; Stefanini, M.; Rufato, L. Dinâmica temporal do míldio da videira em variedades Piwi na região de San Michele all’Adige, Trentino - Itália. Summa Phytopathologica, v.46, n.3, p.212-220, 2020.

O míldio (Plasmopara viticola) é um fungo biotrófico oomyceto agente causal da principal doença da videira. O objetivo deste trabalho foi avaliar a resposta de nove variedades Piwi (do alemão: "Pilzwiderstandsfähige" = "Resistentes às doenças") com resistência constitutiva ao míldio (P. viticola) e uma variedade vinífera (suscetível) como controle em um vinhedo experimental da Fundazione Edmund Mach (FEM), localizado em San Michele All'Adige na região Trentina da Itália. O vinhedo está conduzido por dez anos sem nenhum tratamento químico e foi avaliado na safra agrícola de 2017. As diferentes variedades foram avaliadas quanto a intensidade de míldio a campo e através da densidade estomática analisada no laboratório de Fitopatologia da FEM. Para avaliação da densidade estomática dez folhas de cada genótipo foram coletadas no estádio fenológico de virada de cor, levadas imediatamente ao laboratório e preparadas para observação em lupa (40x) segundo metodologia de Boso et al. (2). No ensaio a campo as avaliações de incidência e severidade do míldio foram realizadas semanalmente a partir do surgimento dos primeiros sintomas/sinais e as epidemias foram comparadas em relação a: a) início do aparecimento dos sintomas/sinais (IAS); b) tempo para atingir a máxima incidência e severidade da doença (TAMID e TAMSD); c) valor máximo de incidência e severidade $\left(I_{\max }\right.$ e $\left.S_{\max }\right)$ d) área abaixo da curva do progresso da severidade e incidência doença (AACPSD e AACPID). As variedades Piwi apresentaram uma redução média de $93,9 \%$ na severidade do míldio em relação a variedade vinífera Pinot Grigio. Jasmine, Muscaris e Regent, foram as variedades que apresentaram a menor intensidade de míldio a campo na safra 2017 nas condições edafoclimáticas Trentina na Itália. Houve uma relação positiva entre a menor densidade estomática por área foliar com a maior resistência a $P$. viticola a campo. As variedades Piwi surgem como uma opção de cultivo visando a redução do uso de agrotóxicos e um suporte sustentável ao setor viti-enológico Trentino.

Palavras-chave: densidade estomática, variedades resistentes, viticultura Trentina

\section{ABSTRACT}

Bem, B.P.; Bogo, A.; Brighenti, A.F.; Wruz, D.A.; Allebrandt, R.; Stefanini, M.; Rufato, L. Temporal dynamics of grapevine downy mildew in Piwi varieties in San Michele all'Adige Region, Trentino - Italy. Summa Phytopathologica, v.46, n.3, p.212-220, 2020.

Downy mildew (Plasmopara viticola), a biotrophic oomycete, is the causal agent of the main disease of the grapevine. The objective of this study was to evaluate the response of nine Piwi varieties (from the German word: "Pilzwiderstandsfähige" = "Disease-resistant") showing constitutive resistance to downy mildew (P. viticola) and a Vitis vinifera variety (susceptible) as control in an experimental vineyard of Fundazione Edmund Mach (FEM), located in San Michele All'Adige, in the Trentino region of Italy. The vineyard has been conducted for ten years without any chemical treatment and was evaluated in the 2017 crop season. The different varieties were evaluated for downy mildew intensity in the field and stomatal density in the Phytopathology Laboratory of FEM. To evaluate stomatal density, ten leaves of each genotype were collected in the phenological stage véraison, immediately taken to the laboratory and prepared for observation through a magnifying glass (40x), according to Boso et al. (2). In the field trial, mildew incidence and severity assessments were carried out weekly from the beginning of the first symptoms/signs and the epidemics were compared in relation to: a) beginning of symptom/sign appearance (BSA); b) time to reach maximum disease incidence and severity (TRMDI and TRMDS); c) maximum value of disease incidence and severity (Imax and Smax), and d) area under the disease incidence and severity progress curve (AUIDPC and AUSDPC). Piwi varieties presented a mean reduction of $93.9 \%$ in mildew severity in relation to the vine variety 'Pinot Grigio'. The varieties 'Jasmine', 'Muscaris' and 'Regent' showed the lowest intensity of downy mildew infection in the field in the 2017 harvest under the edaphoclimatic conditions of Trentino, Italy. There was a positive relationship between the lowest stomatal density per leaf area and the greatest resistance to $P$. viticola in the field. Piwi varieties appear as a cultivation option with the aim of reducing the use of pesticides and as sustainable support for the Trentino wine industry.

Keywords: stomatal density, resistant varieties, Trentino viticulture 
Na região Trentina da Itália a principal doença fúngica que ataca as videiras é o míldio Plasmopara viticola (Berk. \& Curt) Berl. \& de Toni, juntamente com oídio Uncinula necator (Schwein.) Burril), o que demanda um programa de melhoramento da Fundazione Edmund Mach (FEM) em desenvolver novas variedades e avaliar variedades já desenvolvidas em outros centros de pesquisa Europeu que apresentem resistência genética a estes patógenos. As variedades clássicas para vinho, uva de mesa e uva passa, da espécie Vitis vinifera L. amplamente distribuídas na Europa e Ásia Menor, são suscetíveis ao míldio (9). O oomiceto infecta todas as partes verdes da videira, folhas e cachos em particular (13). Em condições ambientais favoráveis (umidade relativa do ar acima de $90 \%$ e molhamento foliar superior a duas horas), no desenvolvimento da videira ocorrem numerosos ciclos de infecção, que são responsáveis pela redução da produção, tanto quantitativa como qualitativa (25).

O controle do míldio geralmente requer o uso excessivo de fungicidas, especialmente em regiões de clima temperado chuvoso, para prevenir epidemias e obter uvas de qualidade aceitável. Para se ter uma viticultura economica e ambientalmente sustentável é necessário o uso racional de fungicidas. Uma solução é o uso de novas variedades com resistência à doença através do cruzamento de espécie $V$. vinifera com espécies de Vitis com resistência genética.

Híbridos resistentes ou parcialmente resistentes têm potencial para reduzir significativamente a aplicação de compostos químicos e, portanto, levar a uma substancial contribuição para a sustentabilidade da viticultura, sendo que os tratamentos podem ser limitados durante safras desfavoráveis (altamente chuvosas) ou estádios fenológicos cruciais (como floração e pegamento de frutos). Muitos avanços foram alcaçados no desenvolvimento de variedades resistentes da videira durante o século 20, onde mais de 6000 híbridos foram registados na Europa. Infelizmente a utilização comercial da maioria dessas variedades não ocorreu, devido a baixa qualidade do vinho ou outros fatores (17). Apesar das evidências de que a resistência a doenças não significa necessariamente uma redução na qualidade do vinho em certas variedades, elas não foram aceitas no mercado Europeu (26). No entanto, variedades recém-criadas que apresentaram resistência a doenças no campo e alta qualidade do vinho tem sido aceitas no mercado na última década, particularmente fora da Europa. Ainda assim, essas novas variedades tolerantes ou resistentes produzem um vinho com algumas características que não são apreciadas pelos consumidores Europeus (12). Porém degustações mais recentes, demonstraram que é possível elaborar vinho com estas variedades com qualidade semelhante àquele de variedades tradicionais (1). $\mathrm{O}$ recente registro de vinte variedades híbridas de videira (como Regent, Bronner e Solaris) no Catálogo Nacional de Variedades Viníferas da Itália (Catalogo Viti) pode sinalizar um novo reconhecimento de tais estudos e um movimento em direção à aceitação de variedades não tradicionais na Europa.

O presente trabalho visou avaliar a variabilidade fenotípica de variedades de videira Piwi na infecção por $P$. viticola em condições naturais, em um vinhedo experimental não tratado pertencente a FEM e sob a densidade estómatica das folhas. Esse experimento buscou demonstrar o comportamento das variedades resistentes nas condições edafoclimáticas na região Trentina da Itália, as quais são distintas das condições encontradas no sul do Brasil, como um ponto inicial de comparação para melhoristas e pesquisadores que pretendem desenvolver o uso dessas variedades através da avaliação dos materiais nas diferentes regiões vitícolas do mundo, tanto no hemisfério norte como no sul.

\section{MATERIAL E MÉTODOS}

\section{Material Vegetativo}

O material vegetativo avaliado consistiu de nove variedades de videira (Bronner, Muscaris, Solaris, Jasmine, Pölöskei Muskotály, Bianca, Cabernet Cortis, Cabernet Carbon e Regent) originadas de várias instituições de pesquisa europeias e a variedade Pinot Grigio ( $V$. vinifera), como controle positivo. Todos os híbridos e o controle positivo foram cultivados em um vinhedo não tratado com fungicidas pertencente a Fondazione Edmund Mach FEM (46 11 '41,46"N, $11^{\circ} 08^{\prime} 04,41^{\prime}$ 'L, altitude 223m), localizado em San Michele All'Adige, Província de Trento, denominado "Campo Inferno" (Figura 1). Cada genótipo foi representado por um único lote de 25 plantas cultivado e manejado desde 2009 usando o método de poda em Guyot, com sistema de condução espaldeira com densidade de plantio de 6250 plantas.ha $^{-1}$ $(0,8 \mathrm{~m} \times 2,0 \mathrm{~m})$. Neste vinhedo não é realizado nenhum tratamento fitossanitário e o isolamento geográfico parcial com outras parcelas de manejo convencional, torna a área imune a qualquer efeito de deriva.

\section{Monitoramento Climático}

O monitoramento das condições climáticas foi feito com base nos dados coletados na Estação Meteorológica Automática localizada no vinhedo. Os dados coletados foram inseridos no banco de dados do setor de Metereologia da FEM. Os elementos climáticos foram: temperatura média do $\operatorname{ar}\left({ }^{\circ} \mathrm{C}\right)$, umidade relativa do $\operatorname{ar}(\%)$ e precipitação pluvial (mm).

\section{Avaliação do míldio}

A incidência e a severidade do míldio foram quantificadas quinzenalmente à partir do início do aparecimento dos sintomas/sinais

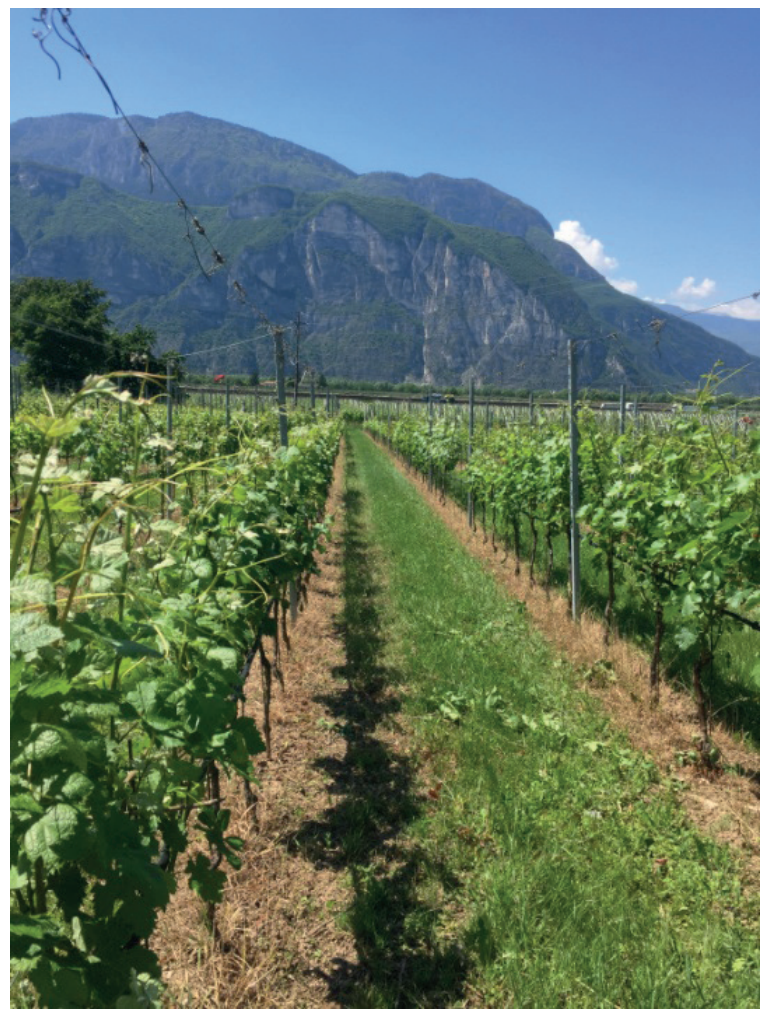

Figura 1. Detalhe do vinhedo experimental "Campo Inferno" da Fundazione Edmund Mach, Trentino - Itália. 2017. Fonte: Elaborada pela autora, 2019. 
até a colheita, em folhas distribuídas em dois ramos medianos da planta com cinco repetições por tratamento. A incidência foi calculada pela porcentagem das folhas com pelo menos uma lesão em relação ao número total avaliado. Para avaliação da severidade foi utilizada a escala diagramática de Buffara et al. (3).

\section{Avaliação da densidade estomática}

Para a quantificação da densidade estomática nos diferentes genótipos foi realizada a contagem do número de estômatos por milímetro quadrado de folha em cada variedade segundo metodologia proposta por Boso et al. (2). A coleta das folhas ocorreu no estádio fenológico de mudança de cor das bagas, durante o período da maturação para todas as variedades. Foram coletadas dez folhas por tratamento/ variedade sendo escolhidas folhas sadias. As folhas coletadas foram imediatamente acondicionadas em sacos plásticos e conduzidas para a preparação de lâminas no Laboratório de Fitopatologia da Fundazione Edmund Mach. Foram preparadas três lâminas por folha, em um total de nove repetições por tratamento/variedade. O delineamento experimental adotado foi o inteiramente casualizado. As lâminas para a contagem dos estômatos foram preparadas com a aplicação de esmalte comercial incolor na superfície foliar abaxial. Após a secagem do esmalte, uma fita adesiva foi colocada na mesma superfície e retirada em seguida com um "negativo" da epiderme foliar, que foi separada da fita com o auxílio de uma pinça. O "negativo" da epiderme foi então colocado sobre uma lâmina de microscopia para visualização ao microscópio, conforme metodologia de Boso et. al. (2). Para a visualização, utilizouse um computador acoplado a lupa estereoscópica com um programa para contagem em magnificação de 40x. Para transformação dos dados por área foi realizado a contagem dos estômatos e dividido pela área observada na lupa que foi de $0,250 \mathrm{~mm}^{2}$, sendo aplicada a fórmula: $\mathrm{D}=$ número de estômatos $/ 0,250 \mathrm{~mm}^{2}$ onde, $\mathrm{D}$ representa a densidade estomática.

\section{Análise estatística}

Através dos dados obtidos a doença foi comparada através do início do aparecimento dos sintomas (IAS); tempo para atingir a máxima incidência e severidade da doença (TAMID e TAMSD); valores máximos de incidência e severidade e Área Abaixo da Curva de Progresso da Doença (AACPD). Para o cálculo da AACPD foi utilizado a seguinte fórmula: $\mathrm{AACPD}=\Sigma((\mathrm{Yi}+\mathrm{Yi}+1) / 2)(\mathrm{ti}+1-\mathrm{ti})$, onde $\mathrm{Y}$ representa a intensidade da doença, t o tempo e i o número de avaliações no tempo (10). As médias dos dados obtidos foram submetidas à análise de variância (ANOVA) e a detecção de diferenças significativas entre os tratamentos foi obtida através do teste Tukey $(\mathrm{P}<0,05)$, através do programa estatístico Statistical Analysis System $\left(\mathrm{SAS}^{\circledR}\right)$.

\section{RESULTADOS}

\section{Avaliação do míldio}

Ocorreram condições para ocorrência do míldio na safra 2017. No período de crescimento vegetativo do vinhedo (de maio à outubro) a temperatura média foi de $19,6^{\circ} \mathrm{C}$. A precipitação pluvial acumulada nesse período foi de $649,2 \mathrm{~mm}$. Nesse período o maior acúmulo de chuva mensal foi no mês de agosto (179,6 mm) (Figura 2). A umidade relativa do ar, média da safra, foi de $48,1 \%$. Apesar de não ser o objetivo deste trabalho, observou-se a presença de oídio nas variedades avaliadas, o que mostra que as condições climáticas locais, principalmente a baixa umidade relativa do ar, permitiram o desenvolvimento desta doença no ciclo 2017 (Figura 2).

As variáveis de quantificação da epidemia estão apresentadas na Tabela 1. A variedade vinífera Pinot Grigio apresentou maior intensidade do míldio em relação as variedades PIWI na safra 2017.

Dentre as variedades de uvas brancas a Bronner mostrou atraso no início do aparecimento dos sintomas/sinais (IAS) em relação as demais variedades, com média de 31 dias (Tabela 1). A variedade avaliada como controle positivo (Pinot Grigio) apresentou a maior antecipação do IAS com média de 16 dias, diferindo estatisticamente das demais (Muscaris, Solaris, Pölöskei Muskotály, Jasmine e Bianca) que mostraram um valor intermediário para o início do aparecimento

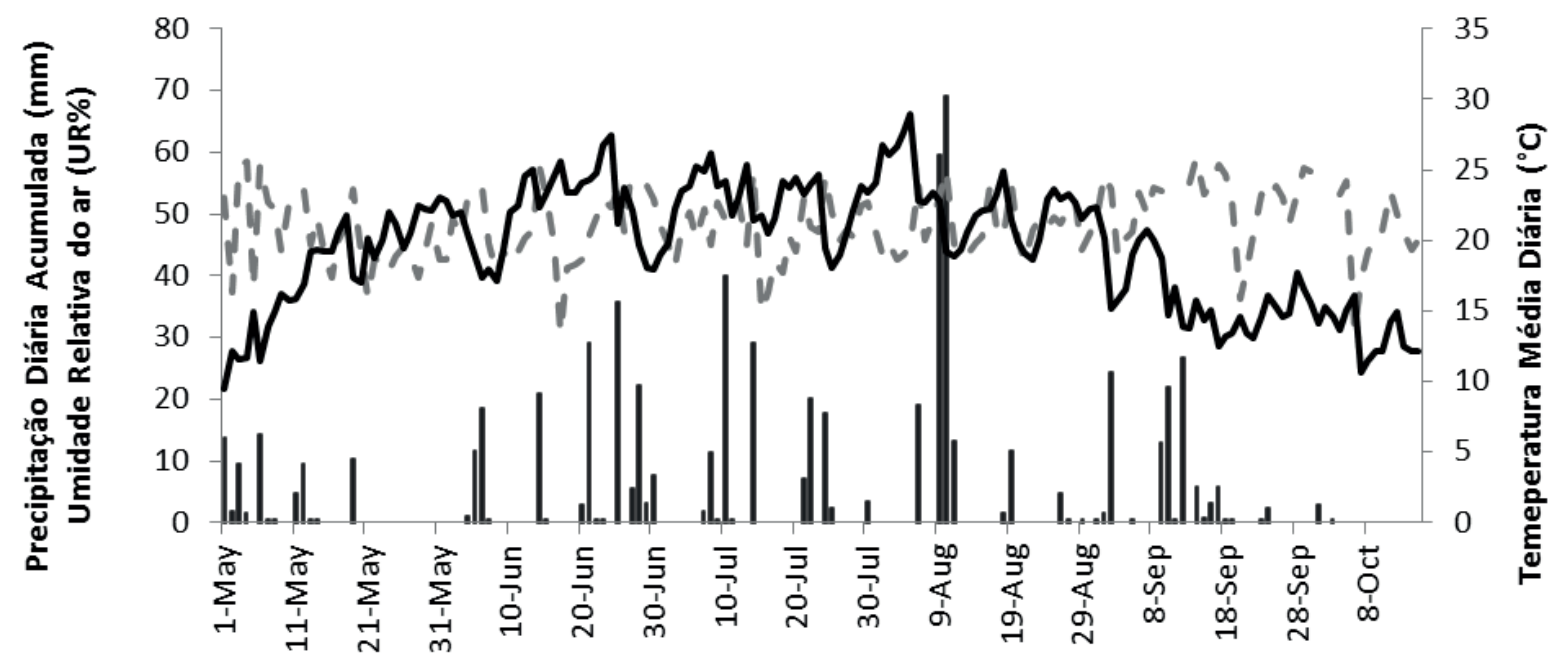

$\square$ Precipitação Diária Acumulada (
$\longrightarrow$ Temperatura Média Diária $\left({ }^{\circ} \mathrm{C}\right)$

- Umidade Relativa do Ar (\%)

Figura 2. Precipitação pluvial acumulada (mm), umidade relativa do ar (\%) e temperatura média mensal ( $\left.{ }^{\circ} \mathrm{C}\right)$ de San Michele All'Adige, Província de Trento, na safra 2017. Fonte: Fundazione Edmund Mach, 2017. 
dos sintomas de 22,3 dias. Para as variedades de bagas tintas observase a mesma tendência, Pinot Grigio com a maior antecipação do IAS, diferindo estatisticamente das demais variedades. Cabernet Carbon e Regent mostraram valores intermediários (27 dias) e Cabernet Cortis mostrou-se a mais eficiente atrasando o IAS para 35 dias.

A variedade que apresentou a menor incidência máxima foi a Muscaris entre as brancas e Regent entre as tintas. O tempo para atingir a máxima incidência da doença foi antecipado em 29 e 33 dias na variedade vinífera controle em relação as demais variedades brancas e tintas, respectivamente.

As variedades avaliadas não diferiram no tempo para atingir a máxima severidade da doença (TAMSD), que ocorreu em média 84 dias após a primeira avaliação (correspondendo a meados do mês de agosto de 2017). A severidade máxima foi superior na variedade vinífera (84), e os menores valores observados foram nas variedades Muscaris(1,4) e Jasmine $(5,3)$ dentre as brancas, mas que não diferiram estatisticamente de Solaris e Bronner. Entre as tintas os menores valores de severidade máxima foram observados nas variedades Cabernet Cortis e Cabernet Carbon.

Houve diferenças significativas entre as variedades em relação a área abaixo da curva de progresso da severidade e incidência (intensidade) da doença (AACPSD e AACPID). A intensidade do míldio foi maior na variedade vinífera Pinot Grigio em relação as variedades Piwi, na safra 2017 (Tabela 1). Para AACPID o menor valor observado entre as variedades brancas foi para variedade Muscaris que não diferiu estatisticamente da variedade Jasmine, as quais apresentaram uma AACPID em média 58,4\% menor que a variedade controle. As variedades Bianca, Solaris, Bronner e Pölöskei Muskotály apresentaram valores intermediários de evolução da incidência ao longo do tempo. Em relação as variedades tintas as Piwi Cabernet Cortis, Cabernet Carbon e Regent apresentaram uma redução média de 40,5 \% na AACPID diferindo estatisticamente da variedade Pinot Grigio.

Em relação ao parâmetro epidemiológico AACPSD, nas variedades brancas seguiu a mesma tendência do que foi observado na AACPID, onde Muscaris e Jasmine apresentaram a maior redução no progresso da severidade do míldio, reduzindo $99,4 \%$ a doença em relação a variedade vinífera. Nas variedades tintas Regent mostrou a menor AACPSD (29,8), não diferindo estatisticamente de Cabernet Cortis e Cabernet Carbon, sendo que apresentaram em média uma redução de $94,4 \%$ do míldio em relação a Pinot Grigio.

Em relação ao desenvolvimento temporal da doença observa-se nos gráficos que na safra avaliada as variedades PIWI, tanto brancas como tintas, apresentaram uma menor AACPS e da incidência da doença em relação a variedade controle positivo vinífera Pinot Grigio (Figuras 3 e 4). O aumento significativo da severidade da doença ocorreu aos 56 dias após a primeira avaliação (DAPA) para a safra 2017. Esse período corresponde a data de 19 de julho 2017. Em relação a AACPID observase que, principalmente nas variedades tintas, ao final da avaliação as variedades PIWI e a vinífera apresentam valores muito próximos. Esse fato está relacionado ao parâmetro incidência da doença apenas avaliar a presença ou ausência do patógeno, sendo que através das curvas da severidade do míldio o efeito da resistência constitutiva das variedades PIWI pode ser detectado de melhor forma.

Tabela 1. Início do aparecimento dos sintomas/sinais (IAS) (dias), incidência máxima (Imax) média (\%), tempo médio para atingir máxima incidência e severidade da doença (TAMID e TAMSD) (dias), severidade máxima (Smax) média (\%), área abaixo da curva do progresso da incidência (AACPID) e severidade (AACPSD) do míldio da videira a campo em diferentes variedades, no ciclo 2017

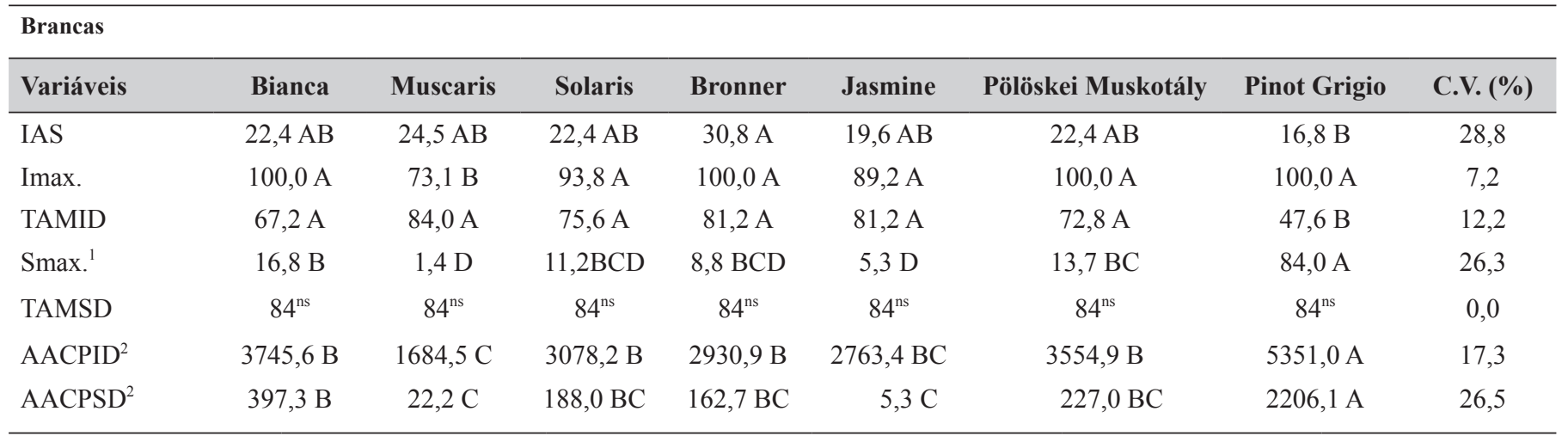

Tintas

\begin{tabular}{|c|c|c|c|c|c|}
\hline Variáveis & Cabernet Carbon & Regent & Cabernet Cortis & Pinot Grigio & C.V. (\%) \\
\hline Imax. & 100,0 & $36,0 \mathrm{~B}$ & $97,3 \mathrm{~A}$ & $100,0 \mathrm{~A}$ & 9,8 \\
\hline TAMID & $78,4 \mathrm{~A}$ & $84,0 \mathrm{~A}$ & $81,2 \mathrm{~A}$ & $47,6 \mathrm{~B}$ & 9,6 \\
\hline Smax. ${ }^{1}$ & $13,8 \mathrm{C}$ & $29,8 \mathrm{~B}$ & 7,4 C & $84,0 \mathrm{~A}$ & 17,4 \\
\hline TAMSD & $84^{\text {ns }}$ & $84^{\mathrm{ns}}$ & $84^{\mathrm{ns}}$ & $84^{\mathrm{ns}}$ & 0,0 \\
\hline $\mathrm{AACPSD}^{2}$ & 219,2 B & 29,8 B & $116,8 \mathrm{~B}$ & $2206,1 \mathrm{~A}$ & 21,9 \\
\hline
\end{tabular}

${ }^{1}$ Estimada pela porcentagem de área foliar lesionada, com o auxílio de escala diagramática, ${ }^{2}$ Calculada por integração trapezoidal conforme Campbell \& Madden (1990), ${ }^{3}$ Médias seguidas da mesma letra maiúscula na linha em cada variedade,não diferem estatisticamente entre si pelo teste Tukey $(\mathrm{P}<0,05)$. 


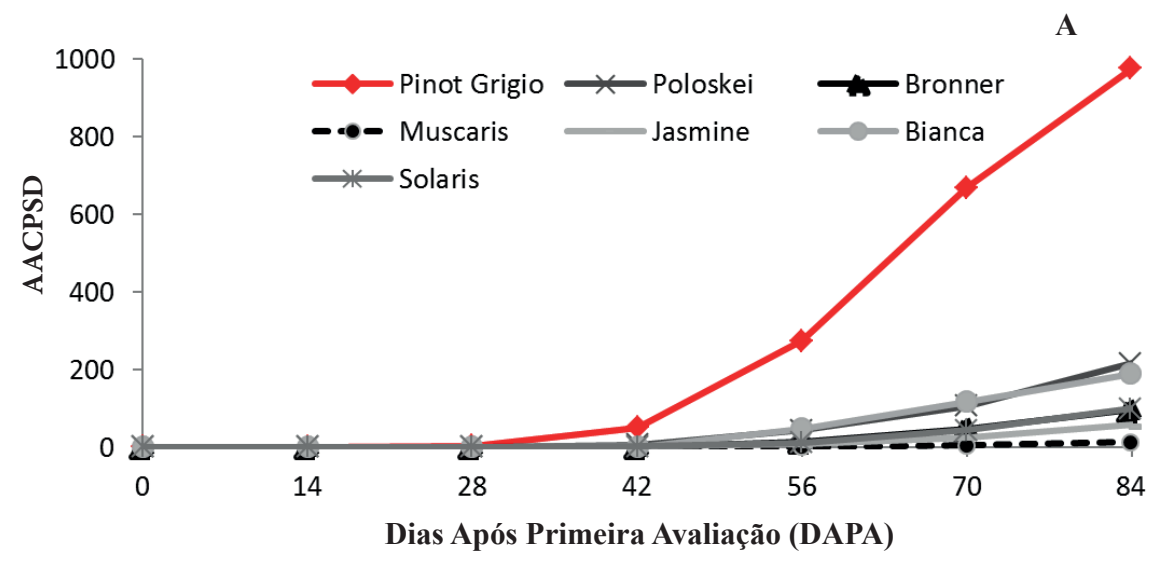

B

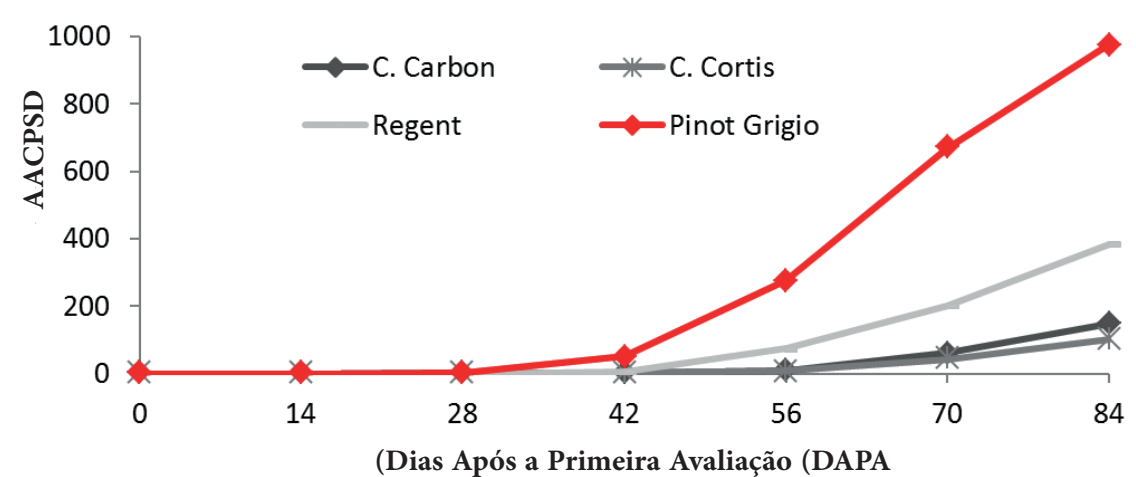

Figura 3. Áreas abaixo da Curva de Progresso da Severidade do míldio a campo, ciclo 2017. A) Variedades Brancas B) Variedades Tintas. Fonte: Elaborada pela autora, 2019.
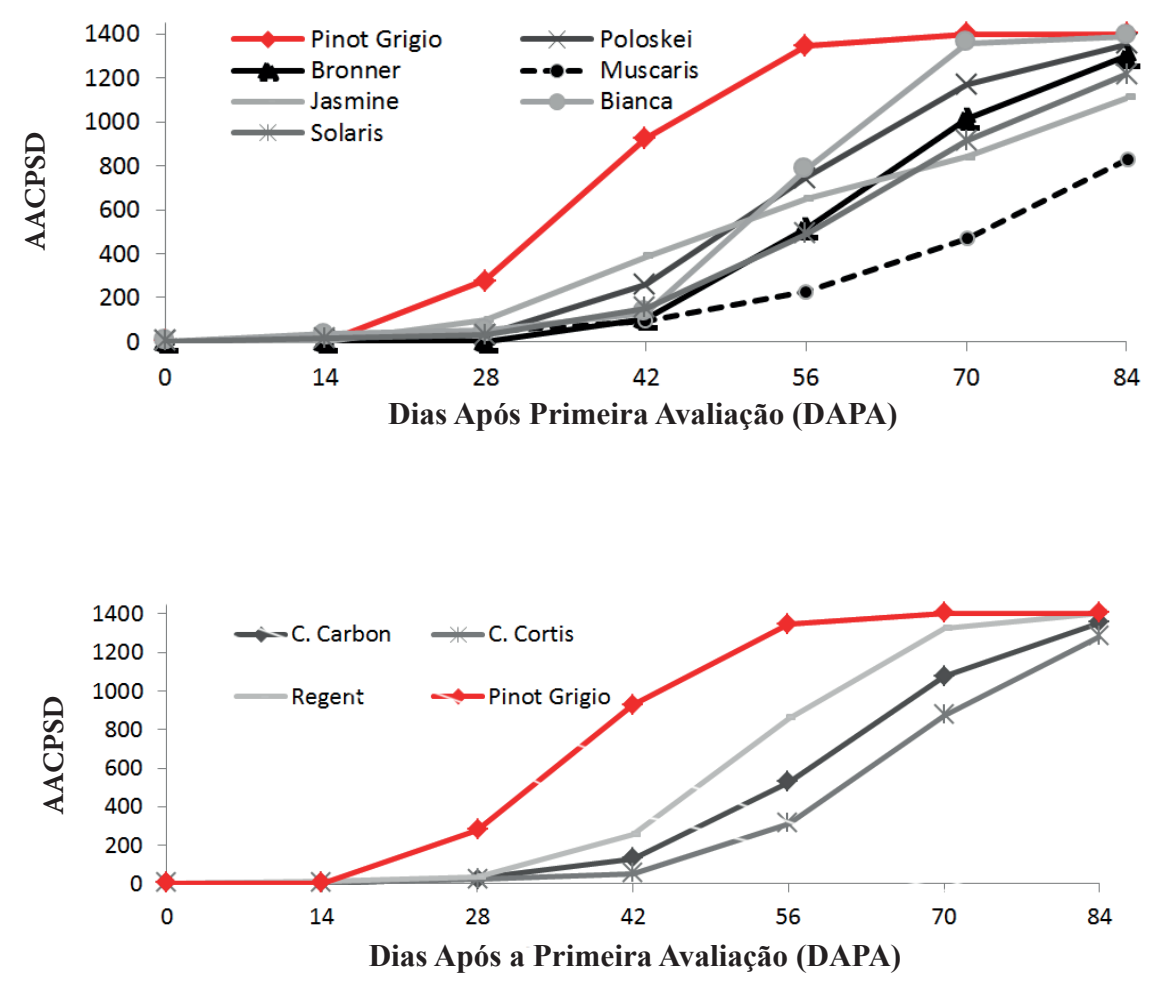

Figura 4. Áreas abaixo da Curva de Progresso da Incidência do míldio a campo, ciclo 2017. A) Variedades Brancas B) Variedades Tintas. 


\section{Avaliação da densidade estomática}

Dentre as variedades brancas, as variedades Piwi Solaris, Pölöskei Muscotály e o controle positivo Pinot Grigio que apresentaram maior suscetibilidade, foram as que apresentaram a maior densidade estomática nas folhas (Figura 5). Da mesma forma, as variedades que mostraram maior resistência ao patógeno, foram aquelas que diferiram estatisticamente apresentando uma menor densidade estomática como Bronner e Jasmine com média de 66,7 estômatos $/ \mathrm{mm}^{2}$ de área foliar (Tabela 2). Esta relação não foi verificada na variedade Bianca, que apresentou uma maior suscetibilidade ao patógeno, porém mostrou uma baixa densidade estomática $\left(66,5\right.$ estômatos $/ \mathrm{mm}^{2}$ de área foliar).

Dentre as variedades tintas observa-se a relação positiva entre a severidade da doença a campo (AACPSD) e a densidade estomática. Regent que mostrou a menor AACPSD apresentou a menor densidade estomática (87 estômatos $/ \mathrm{mm}^{2}$ de área foliar), Cabernet Cortis e C. Carbon valores intermediários (média 98,7 estômatos $/ \mathrm{mm}^{2}$ de área foliar) e a variedade vinífera controle os maiores valores de severidade da doença bem como a maior densidade estomática (114, 5 estômatos/ $\mathrm{mm}^{2}$ de área foliar) (Figura 6).
Tabela 2. Densidade estomática em folhas de diferentes variedades PIWI e viníferas

\begin{tabular}{cc}
\hline \multicolumn{2}{c}{ Densidade Estomática } \\
\hline Brancas & Número de estômatos $/ \mathbf{m m}^{\mathbf{2}}$ \\
\hline Solaris & $131,0 \mathrm{~A}^{1}$ \\
Pölöskei Muskotály & $121,7 \mathrm{~A}$ \\
Pinot Grigio & $114,5 \mathrm{~A}$ \\
Bronner & $76,0 \mathrm{~B}$ \\
Bianca & $66,5 \mathrm{~B}$ \\
Jasmine & $57,5 \mathrm{~B}$ \\
\hline C.V. (\%) & 21,7 \\
\hline Tintas & Número de estômatos $\left(\mathbf{~ m m}^{\mathbf{2}}\right)$ \\
\hline Pinot Grigio & $114,5 \mathrm{~A} 1$ \\
C. Cortis & $103,0 \mathrm{AB}$ \\
C. Carbon & $94,5 \mathrm{AB}$ \\
Regent & $87,0 \mathrm{~B}$ \\
\hline C.V. (\%) & 18,2 \\
\hline
\end{tabular}

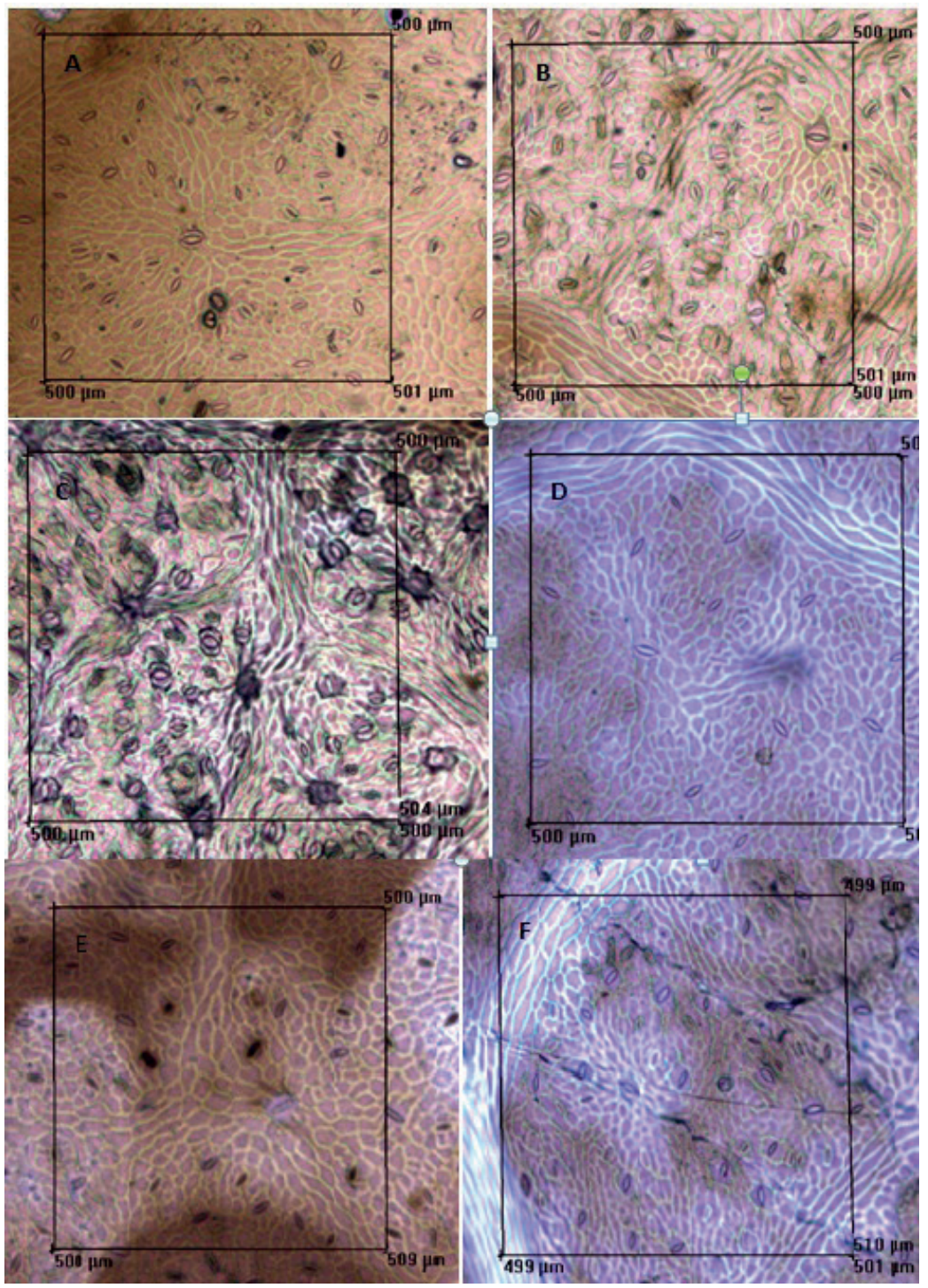

Figura 5. Estômatos por $0,250 \mathrm{~mm}^{2}$ de área abaxial foliar de diferentes variedades de videira através de programa de computação acoplado a lupa estereoscópica com lente objetiva de 40x. Variedades brancas: A) Pinot Grigio B) Poloskei C) Solaris D) Jasmine E) Bianca F) Bronner, em Fundazione Edmund Mach, Trentino, Itália. Fonte: Elaborada pela autora, 2019. 


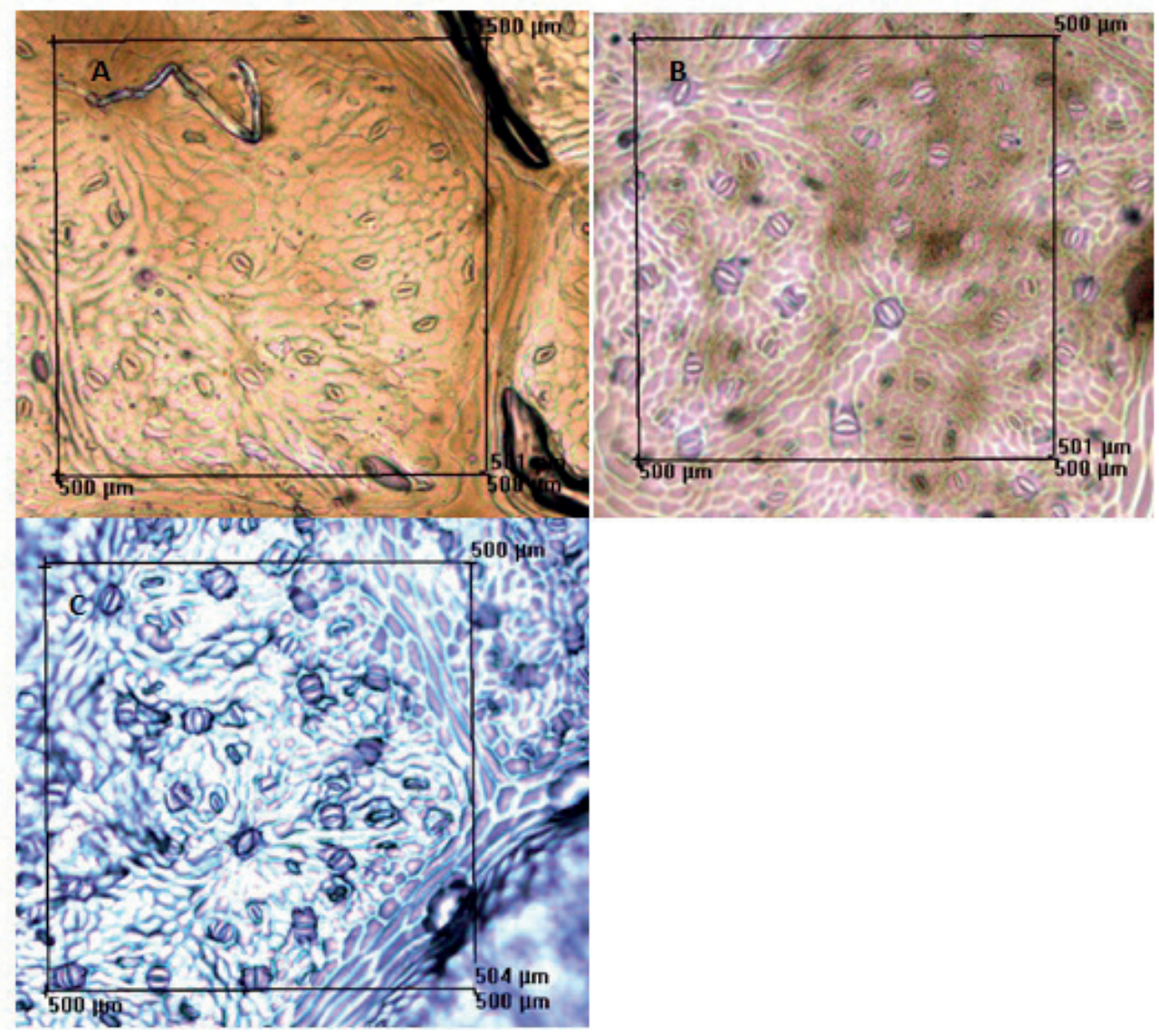

Figura 6. Estômatos por $0,250 \mathrm{~mm}^{2}$ de área abaxial foliar de diferentes variedades de videira através de programa de computação acoplado a lupa estereoscópica com lente objetiva de 40x. Variedades brancas: A) Regent B) Cabernet Carbon C) Cabernet Cortis Fundazione Edmund Mach, Trentino, Itália. Fonte: Elaborada pela autora, 2019.

\section{DISCUSSÃO}

\section{Avaliação do míldio a campo}

Observa-se que na safra avaliada houveram condições ambientais favoráveis a ocorrência do míldio da videira. Segundo Garrido \& Sônego (10) as condições climáticas para o desenvolvimento da doença são temperaturas entre $18{ }^{\circ} \mathrm{C}$ e $25^{\circ} \mathrm{C}$ e umidade relativa do ar acima de $60 \%$. Todos os fatores que contribuem para aumentar o teor de água no solo, ar e planta favorecem o desenvolvimento do míldio da videira. A temperatura exerce papel moderador, paralisando o progresso da doença. Dificilmente ocorre infecção se a umidade do ar for inferior a 75\%; porém, ela será mais severa quando a duração do período de molhamento foliar for superior a três horas. Na safra avaliada, quando compara-se o desenvolvimento do míldio nas condições climáticas do Brasil (7) observa-se que a umidade relativa do ar teve grande influência sobre o progresso da doença nos diferentes países. No Sul do Brasil as médias históricas de precipitação pluvial média e a umidade relativa do ar média são de $665,9 \mathrm{~mm}$ e $82,6 \%$, respectivamente. Na Itália essas mesmas variáveis corresponderam a valores médios de $649,2 \mathrm{~mm}$ e $48,1 \%$, na safra avaliada de 2017 . Verifica-se que a precipitação pluvial média no período de crescimento vegetativo do vinhedo é muito próxima, mas a média da umidade relativa do ar é muito superior no Brasil, sendo que o clima na região Trentina é mais seco. Essa condição resulta na redução do período de molhamento foliar, apesar de haver precipitação pluvial, a água livre evapora rapidamente pela condição de baixa umidade relativa do ar. Comprovadamente a baixa umidade relativa do ar e a ausência de água livre na folha são fatores limitante para sucesso da infecção do patógeno. Por outro lado, essas condições favorecem o desenvolvimento do oídio, como foi observado na região Trentina e não no Sul do Brasil, durante as safras avaliadas.

Em relação ao desenvolvimento do míldio nas diferentes variedades brancas pode-se confirmar que Muscaris e Jasmine mostraram o maior grau de resistência. A variedade Jasmine foi incluída no estudo por ser relatada como uma das variedades mais resistentes dentro do programa de melhoramento genético da FEM, utilizada como fonte de resistência para os cruzamentos por possuir uma variabilidade no gene de resistência ao míldio, através do locus Rpv-12, o que foi comprovado a campo na safra 2017. Bronner, Pölöskei Muscotály e Solaris apresentaram valores intermediários. Os resultados encontrados para resistência foliar das variedades Piwi Bronner, Muscaris, Pölöskei Muscotály e Solaris estão de acordo com o observado por outros autores em relação ao nível de resistência ao míldio $(6,21,22,29)$.

A variedade Bianca apresentou maior suscetibilidade diferindo estatisticamente apenas da vinífera Pinot Grigio. Destaca-se que Bianca se mostrou uma variedade altamente resistente nas avaliações no sul do Brasil em São Joaquim - SC (8) com a maior expressão de reação de hipersensibilidade (HR), na região Trentina observou-se o oposto, com os valores mais altos de intensidade da doença dentre as variedades PIWI avaliadas. Este fato pode estar relacionado ao estudo recente que comprovou a superação da resistência da variedade 'Bianca' 
por um isolado tcheco de P. viticola (19). Quanto a resistência da variedade Bianca no continente Europeu, há divergências nos resultados obtidos até o momento. Em estudos mais antigos, observou-se níveis de resistência foliar ao míldio pela variedade Bianca $(21,29)$. Por outro lado, estudos recentes mostraram uma maior suscetibilidade da variedade Bianca (27).

Entre as variedades tintas de resistência intermediária (Cabernet Cortis e Cabernet Carbon) não é possível fazer uma comparação válida com a literatura existente. Porém apresentaram alta resistência a campo, o que indica que podem ser utilizadas inclusive em programas futuros de melhoramento no Brasil. A variedade Regent comprovou seu alto grau de resistência a campo na safra avaliada de 2017, através do gene Rpv3-1, como demostrado em outros estudos, e na safra 2015/16, avaliada em São Joaquim - SC (8). Basler e Pfenninger (1) avaliando a resistência de diferentes genótipos ao míldio na Suíça Oriental, especialmente no lago de Zurique (condições altamente favoráveis ao desenvolvimento da doença, como precipitações médias de $1367 \mathrm{~mm}$ ao ano) listaram 20 variedades resistentes, entre elas a Regent, como uma opção para o cultivo orgânico, visando a redução do uso de fungicidas, principalmente a base de cobre, o que vem sendo uma busca atual e constante pelos viticultores e consumidores nos países Europeus.

\section{Avaliação da densidade estomática}

Da mesma forma que já comprovado por por outros autores (15), observa-se no presente estudo que há uma relação positiva entre maior densidade estomática por área foliar e suscetibilidade ao míldio. Apesar da avaliação dos estômatos ser difícil devido a grande variação de fatores que podem alterar o número e tamanho destas estruturas (2), as variedades que se mostraram resistentes a campo como Jasmine, Bronner e Regent também foram aquelas que apresentaram a menor densidade estomática.

A média da densidade estomática observada na região Trentina entre variedades Piwi e vinífera foi de 100,4 estômatos. $\mathrm{mm}^{2}$ de área foliar, esses dados estão de acordo com relatos de outros autores (24, 28) que indicam condições ambientais (chuva, concentração de $\mathrm{CO}_{2}$ atmosférico, intensidade da luz, temperatura do ar), fotoperíodo e manejo podem influenciar na densidade estomática. Porém verificouse a relação entre maior resistência à doença com a menor densidade estomática.

Existem controvérsias se a densidade estomática é geneticamente pré-determinada ou se pode variar de acordo com condições ambientais como intensidade de luz, umidade, temperatura do ar ou temperatura do solo, etc. Palliotti et al. (18) defendem que a densidade estomática é uma condição estritamente genética, enquanto outros autores (5, $14,20)$ argumentam que condições fenotípicas contribuem para esta característica morfológica das folhas de videiras. Alguns autores $(11,20)$, sugerem que tanto fatores ambientais bem como diferenças genéticas atuam conjuntamente neste fator. Segundo Meisel et al. (16), as plantas têm uma sistema de "percepção", que lhes permite captar ondas de luz através de fotorreceptores especializados. Estes iniciam eventos bioquímicos que regulam a atividade do genoma, permitindo que ocorram respostas aos estímulos ambientais. Sugano et al. (23) relatou recentemente que as células do mesófilo de folhas de Arabidopsis imaturas expressam um fator de sinalização intercelular que interage com células epidérmicas para influenciar a densidade estomática. Pode ser, portanto, que essas células fotossintéticas, e não as células epidérmicas, em última análise, regulem essa variável.

No presente estudo verificou-se que a maior parte das variedades manteve o mesmo padrão de densidade estomática quando comparado com outros estudos, possivelmente ligados a fatores genéticos, e algumas variações entre as variedades que ocorreram devem estar ligadas aos fatores ambientais locais diversos.

As variedades PIWI avaliadas, principalmente Jasmine, Muscaris e Regent, mostraram menor intensidade de míldio a campo em comparação com a variedade vinífera Pinot Grigio na safra 2017 nas condições edafoclimáticas da Região de Trento. Houve uma relação positiva entre a menor densidade estomática por área foliar com a maior resistência a $P$. viticola .

\section{REFERÊNCIAS}

1. Basler, P.; Pfenninger, H. Disease-resistant cultivars as a solution for organic viticulture. ISHS Acta Horticulturae, Hungary, v. 603, p.681-685, 2003.

2. Boso, S.; Gago, P.; Alonso-Villaverde, V.; Santiago, J.L.; Martinez, M.C. Density and size of stomata in the leaves of different hybrids (Vitis sp. and Vitis vinifera varieties. Vitis, Geilweilerhof, v.55, p.17-22, 2016.

3. 0.383Buffara, C.R.C. ; Angelotti, F.; Vieira, F.A. ; Bogo, A.; Tessmann, D.J.; De Bem, B.P. Elaboration and validation of a diagrammatic scale to assess downy mildew severity in grapevine. Ciência Rural, Santa Maria, v.44, n.8, p.1384-1391, 2014.

4. Campbell, C.L.; Madden, L.V. Introduction to plant disease epidemiology. New York: Wiley, 1990. 560p.

5. Casson, S.; Gray, J. E. Influence of environmental factors on stomatal development. New Phytologist, British, 178, 9-23. 2008.

6. Cindric, P.; Korac, N.; Kovac, V. Grape breeding for resistance. ISHS Acta Horticolturae, Hungary, v. 603, p.385-391, 2003.

7. De Bem, B. P.; Bogo, A.; Everhart, S.; Casa, R.T.; Gonçalves, M.J.; Marcon Filho, J.L.; Cunha, I.C. Effect of Y-trellis and vertical shoot positioning training systems on downy mildew and botrytis bunch rot of grape in highlands of southern Brazil. Scientia Horticulturae, Amsterdam, v.185, p.162-166, 2015.

8. De Bem, B.P.; Brighenti, E.; Bonin, B.; Allembrandt, R.; Araújo, L.; Brighenti, A.F.; Bogo, A. Downy mildew intensity in tolerant grapes varieties in highlands of southern Brazil. BIO Web of Conferences, Bento Gonçalves, v.7, p.01015. 2016.

9. Deglène-Benbrahim, L.; Wiedemann-Merdinoglu, S.; Merdinoglu, D.; Walter, B. Evaluation of downy mildew resistance in grapevine by leaf discs bioassay with in vitro-and greenhouse-grown plants. American Journal of Enology and Viticulture, Davis, v.61, p.521-528. 2010.

10. Garrido, L.R.; Sônego, O.R.; Valdebenito-Sanchueza, R.M. Controle racional de doenças da videira e da macieira. In: Stadnik, M.J.; Talamini, V. Manejo ecológico de doenças de plantas. Florianópolis, p.221-244, 2004.

11. Gómes-Del-Campo, M.; Ruiz, C.; Baeza, P.; Lissarrague, J.R. Drought adaptation strategies of four grapevine cultivars (Vitis vinifera L.): modification of the properties of the leaf area. Journal Internacional des Sciences de la Vigne et du Vin, Paris, v.37, p.131-143. 2003.

12. Guedes de Pinho, P.; Bertrand, A. Analytical determination of furaneol (2,5-dimethyl1-4-hydroxy-3(2H)-furanone). Application to differentiation of white wines from hybrid and various Vitis vinifera cultivars. American Journal of Enology and Viticulture, Davis, v.46, p.181-186, 1995.

13. Ingram, D.S. Physiology and biochemistry of hostparasite interaction. In: Spencer, D.M (ed.). The Downy mildews. London: Academic Press, p.143-163. 1981.

14. Lake, J. A.; Quick, W. P.; Beerling, D. J.; Woodward, F. I. Plant development. Signals from mature to new leaves. Nature, London, v.411, p.154. 2001.

15. Lu, J.; Zhang, Y.; Wang, J. Genetic variation and mechanism of host resistance to Downy Mildew disease among oriental Vitis species. In: International Workshop of Grapevine Downy and Powdery Mildew, 6, 2010. Bordeaux, France. Proceedings. Ed. INRA Bordeaux-Aquitaine, 2010.

16. Meisel L.; Urbina, D.; Pinto, M. Fotorreceptores y respuestas de plantas a señales lumínicas. In: Fisiología vegetal. La Serena: Ediciones Universidad de La Serena, p. 1-10, 2011.

17. Pacifico, D.; Gaiotti, F.; Giusti, M.; Tomasi, D. Performance of interspecific grapevine varieties in north - east Italy. Agricultural Sciences, Cambridge, v. 4, p.91-101, 2013.

18. Palliotti, A.; Cartechini, A.; Ferranti, F. Morpho-anatomical and physiological characteristics of primary and lateral shoot leaves of Cabernet Franc 
and Trebbiano Toscano grapevines under two irradiance regimes. American Journal of Enology and Viticulture, Davis, v.51, p.122-130. 2000.

19. Peressotti, E.; S., Wiedemann-Merdinoglu, S.; Delmotte, F.; Bellin, D.; Di Gaspero, G.;Testolin, R.; Merdinoglu, D.;Mestre, P. Breakdown of resistance to grapevine downy mildew upon limited deployment of a resistant variety. BMC Plant Biology, London, v. 10, p.147, 2010.

20. Rogiers, S. Y.; Hardie, W. J.; Smith, J. P. Stomatal density of grapevine leaves (Vitis vinifera $\mathrm{L}$.) responds to soil temperature and atmospheric carbon dioxide. Australian Journal of Grape and Wine Research, Australia, v. 17, p.147-152. 2011.

21. Schwab, A.L.; Knott, R.; Schottdorf, W. Results from new fungus-tolerant grapevine varieties for organic viticulture. In: 6th International Congress on Organic Viticulture, Switzerland. Proceedings, Basel: Helga Willer, Urs Meier. Switzerland. p. 26-26. 2000.

22. Spring, J.L. Premières expériences avec les cépages interspécifiques Merzling, Johanniter, Bronner et Solaris en Suisse romande. Revue Suisse de Viticulture Arboriculture Horticulture, França, v. 33, p. 57-64. 2001.

23. Sugano, S. S.; Shimada, T.; Imai, Y.; Okawa, K.; Tamai, A.; Mori, M.; Hara-Nishimura, I. Stomagen positively regulates stomatal density in Arabidopsis. Nature, London, v. 463, p. 241-244. 2010.
24. Tichà, I. Photosynthetic characteristics during ontogenesis of leaves. 7. Stomata density and sizes. Photosynthetica, República Tcheca, v. 16, p. 375-471.1982.

25. Toffolatti, S.L.; Venturini, G.; Maffi, D.; Vercesi, A.; Phenotypic and histochemical traits of the interaction between Plasmopara viticola and resistant or susceptible grapevine varieties. BMC Plant Biology, London, v.12, p.124, 2012.

26. Topfer, R.; Hausmann, L.; Eibach, R. Molecular breeding. In: Zapater, M.M.; Adam-Blondom, A.F.; Kole, C. Genetics, Genomics and Breeding of Grapes. Enfield, NH, USA: Science Publisher, p. 160-185. 2011.

27. Vezzulli, S.; Vecchione, A.; Tefanini, M.; Zulini,L. Downy mildew resistance evaluation in 28 grapevine hybrids promising for breeding programs in Trentino region (Italy). European Journal of Plant Pathology. London, v. 150, p. $485-495,2017$.

28. Wentworth, M.; Murchie, E. H.; Gray, J. E.; Villegas, D.; Pastenes, C.; Pinto, M.; Horton, P. Differential adaptation of two varieties of common bean to abiotic stress. II. Acclimation of photosynthesis. Journal of Experimental Botany.v.57, p. 699-709. 2006

29. Zulini, L.; Vecchione, A.; Antonelli, L.; Stefanini, M. Characteristics of wine and table grapevine hybrids tested for cultivation in Trentino (northern Italy). IOBC/WPRS Bullettin, v.36, p. 215-219. 2008. 